\title{
TITLE:
}

\section{Highly entangled polymer primitive chain network simulations based on dynamic tube dilation}

\section{$\operatorname{AUTHOR}(\mathrm{S})$ :}

Yaoita, T; Isaki, T; Masubuchi, Y; Watanabe, H; lanniruberto, G; Greco, F; Marrucci, G

\section{CITATION:}

Yaoita, T ...[et al]. Highly entangled polymer primitive chain network simulations based on dynamic tube dilation. JOURNAL OF CHEMICAL PHYSICS 2004, 121(24): 12650-12654

\section{ISSUE DATE:}

2004-12-22

URL:

http://hdl.handle.net/2433/50370

\section{RIGHT:}

Copyright 2004 American Institute of Physics. This article may be downloaded for personal use only. Any other use requires prior permission of the author and the American Institute of Physics. 


\title{
Highly entangled polymer primitive chain network simulations based on dynamic tube dilation
}

\author{
Takatoshi Yaoita and Takeharu Isaki \\ Material Science Laboratory, Mitsui Chemicals Inc., Chiba 299-0265, Japan \\ Yuichi Masubuchi ${ }^{\text {a) }}$ \\ Department of Organic and Polymer Materials Chemistry, Tokyo University of Agriculture and Technology, \\ Koganei 184-8588, Japan \\ Hiroshi Watanabe \\ Institute for Chemical Research, Kyoto University, Kyoto 611-0011, Japan \\ Giovanni lanniruberto \\ Dipartimento di Ingegneria Chimica, Università degli studi di Napoli “Federico II," Napoli, Italy \\ Francesco Greco \\ Istituto per i Materiali Compositi e Biomedici-CNR, Piazzale Tecchio 80-80125 Napoli, Italy \\ Giuseppe Marrucci \\ Dipartimento di Ingegneria Chimica, Università degli studi di Napoli "Federico II," Napoli, Italy
}

(Received 2 July 2004; accepted 24 September 2004)

\begin{abstract}
The concept of dynamic tube dilation (DTD) is here used to formulate a new simulation scheme to obtain the linear viscoelastic response of long chains with a large number of entanglements. The new scheme is based on the primitive chain network model previously proposed by some of the authors, and successfully employed to simulate linear and nonlinear behavior of moderately entangled polymers. Scaling laws are generated by the DTD concept, and allow for prediction of the linear response of very long chains on the basis of suitable simulations performed on shorter ones, without introducing adjustable parameters. Tests of the method against existing data for linear monodisperse polyisoprene and polystyrene show good quantitative agreement. (C) 2004 American Institute of Physics. [DOI: 10.1063/1.1819311]
\end{abstract}

\section{INTRODUCTION}

Atomistic simulations of concentrated polymeric liquids are limited by the extremely long relaxation times of these systems. As a consequence, several coarse-grained models have been proposed, and some of them achieved remarkable successes in predicting characteristic features of polymer dynamics. Kremer-Grest simulations ${ }^{1}$ adopt coarse-graining at the level of Kuhn segments, and have been used for shorttime or short-chain dynamics of polymers. Entangled polymer dynamics, however, becomes computationally demanding with that approach, and hence sliplink simulations ${ }^{2-4}$ have been developed. These are based on the reptation model $^{5,6}$ together with extensions that include: contour length fluctuations, ${ }^{6}$ thermal ${ }^{7}$ and convective ${ }^{8}$ constraint release, force balance around entanglements, ${ }^{3,9}$ chemical potential field, ${ }^{10}$ hidden entanglement appearance, ${ }^{11,12}$ etc. Sliplink simulations can quantitatively predict the long time behavior of entangled polymers with several entanglements per chain (up to several tens) insofar as the coarse-graining is augmented to the level of the distance between consecutive entanglements. ${ }^{2-4,10,13}$

However, for highly entangled polymers (say, with 100 entanglements per chain or more), no method has so far been

\footnotetext{
a) Author to whom correspondence should be addressed. Telephone and Fax: +81-42-388-7058. Electronic mail: mas@rheo.chem.tuat.ac.jp
}

proposed that remains within acceptable computational cost. On the other hand, for polymers having a small entanglement molecular weight $M_{e}$, e.g., polyolefins, commercial materials in the molten state commonly fall in the category of highly entangled polymers.

The only way to simulate economically long polymer dynamics appears to be by further coarse-graining, and a possible strategy to achieve such an objective is by using the concept of dynamic tube dilation (DTD). Of course, as all coarse-graining strategies, also the one adopted here is empirical to some extent. DTD was first proposed by Marrucci, ${ }^{14}$ and consists of an effective increase of the entangled network mesh size in the course of time (during relaxation), due to thermal motion of the surrounding chains. Originally, ${ }^{14}$ DTD was proposed for linear polymers, either monodisperse or polydisperse, and it affected both the modulus and the relaxation times. Later DTD was extensively applied by McLeish and co-workers ${ }^{15}$ to branched polymers, especially starlike polymers, where relaxation slows down exponentially from each arm end to the branch point. Recently, in the context of a comparison between dielectric and mechanical relaxation, DTD has again been applied to monodisperse linear polymers by Watanabe, though in a modified form, ${ }^{16}$ i.e., by limiting DTD effects to modulus only, while leaving relaxation times untouched. It is in this latter form that DTD will be used in the present paper. 
The general concept of DTD is that, in the course of relaxation, effective obstacles decrease in number (as if by dilution), and therefore the mesh size of the entangled network effectively increases. This has an obvious effect on modulus, which correspondingly decreases. Consequences on longer relaxation times may or may not be present. The effect on times is certainly present in branched polymers, ${ }^{15}$ and it may be present or not in polydisperse linear polymers. For binary homopolymer blends it has been suggested ${ }^{16,17}$ that the condition for the terminal relaxation time of the long polymer to be affected by DTD is fulfillment of the inequality $M_{L} M_{e}^{2} / M_{S}^{3}>0.5$, where $M_{L}$ and $M_{S}$ are the molar mass of the long and short polymer, respectively. Clearly such an inequality (known as Struglinski and Graessley condition) is never fulfilled in a monodisperse sample, where therefore DTD only affects the modulus.

In the next section we use DTD, in the version where only modulus is affected, to formulate rules by which predictions for very long monodisperse chains can be obtained by simulating short ones. In a following section we perform comparison with existing data.

It is finally to be made clear that the method proposed here only applies for the long-time relaxation, i.e., for the relaxation that takes place after the Rouse time of the chain.

\section{MODEL}

\section{Self-multiscale scheme}

In this section, a scheme to obtain the whole relaxation of very long chains is presented, which is based on the dynamic self-similarity implicit in DTD. In the DTD picture, the primitive chain is progressively coarse-grained in the course of time, and hence the effective number of entanglements per chain decreases. There follows that the relaxation functions of short and long chains can be superimposed by suitable scaling rules for length (and, consequently, modulus) as well as time.

Let us consider a monodisperse polymer with $Z$ primitive-chain segments per chain (corresponding to $Z-1$ entanglements per chain). According to DTD, the effective number $Z^{\prime}$ of subchains decreases with time due to the relaxation of surroundings, and we may write

$$
Z^{\prime}=Z / \beta \quad(\beta>1),
$$

where $\beta$ is the dilation ratio. Change in effective entanglement number has immediate consequences on modulus, as detailed later.

With regard to time, as previously explained we want to maintain unaltered the longest relaxation time $\tau_{d}$ scaling as $M^{\alpha}$, where $M$ is molecular weight, and the exponent $\alpha$ is around 3.5. Since in the simulations the reference time is the relaxation time $\tau_{e}$ of the (undiluted) subchains between consecutive entanglements, we must have

$$
\tau_{d} \approx \tau_{e} Z^{\alpha}=\tau_{e}^{\prime}\left(Z^{\prime}\right)^{\alpha} .
$$

Hence, from Eqs. (1) and (2), we obtain the scaling of dilating time $\tau_{e}^{\prime}$ due to DTD as

$$
\tau_{e}^{\prime}=\beta^{\alpha} \tau_{e} .
$$

It is to be remembered, however, that such scaling can only be applied beyond the Rouse relaxation regime. In other words, we expect that the procedure indicated in this paper, though sufficient in shortening the computation time for long molecules, also pays the price of losing accuracy in the frequency (or time) range corresponding to Rouse relaxation.

With regard to modulus, we recall that the plateau modulus $G_{0}$ is usually written as $G_{0}=\nu \rho R T / M_{e}$, where $M_{e}$ is the entanglement molecular weight and $\nu$ is a theory dependent numerical factor. ${ }^{18}$ Accordingly, the modulus of the dilated network should become $G_{0} / \beta$. However, as specified in the following section, in our simulations end subchains do not contribute to $G_{0}$, and hence the scaling of modulus due to DTD is slightly different from that just indicated.

The scaling rules allow one to obtain the relaxation functions of very long chains from calculations made on short ones. For example, if $\beta$ is set to 2,4 , and 8 , the scaling factors for time are, for $\alpha=3.5, \tau_{e}^{\prime} / \tau_{e} \approx 11.3,128$, and 1450, respectively. This means that relaxation of a very long chain can be simulated while reducing the calculation time by a factor up to more than one thousand (if $\beta=8$ can be justified). In the next sections, we will show one such example.

\section{The primitive chain network model}

To test the idea of the self-multiscale scheme discussed in the previous section, the primitive chain network simulations $^{3}$ were employed in this study. However, the selfmultiscale idea can also be applied to other sliplink models, provided they account for constraint release.

The primitive chain network model consists of a threedimensional (3D) network of primitive chains linked through sliplinks. The dynamics of the network is ruled by a 3D Langevin equation for the network nodes (either sliplinks or chain ends) as well as by a 1D Langevin equation describing monomer sliding along the chain through sliplinks. In the model, all known molecular mechanisms for the dynamics of entangled polymers (reptation, contour length fluctuations, thermal and convective constraint release, force balance on entanglements, and chemical potential gradients) are automatically taken into account. More details on the model can be found in our previous publications. ${ }^{3,10,12,13}$

In the primitive chain network model, due to chain end effects, the plateau modulus of the network depends on $Z$ in the following way: ${ }^{13}$

$$
G_{0}(Z)=\left(1-\frac{3}{Z}\right) G_{0}(\infty) .
$$

Here $G_{0}(\infty)$ is the plateau modulus of very long chains, where end effects become negligible. Hence, the DTD scaling rule for modulus, or stress, becomes (see Appendix)

$$
G_{0}\left(Z^{\prime}\right)=\frac{Z^{\prime}-3}{Z-3} G_{0}(Z) .
$$

Notice that if both $Z$ and $Z^{\prime}$ are large numbers, Eq. (5) reduces to the previously mentioned scaling: $G_{0}\left(Z^{\prime}\right)$ $=G_{0}(Z) / \beta$.

Since $Z$ is the number of primitive-chain segments per chain for the undilated tube, it is obtained, as usual, from 
TABLE I. Characteristics and model parameters of monodisperse polyisoprene and polystyrene.

\begin{tabular}{lcccccc}
\hline \hline \multicolumn{1}{c}{ Sample } & $M_{w} /(\mathrm{g} / \mathrm{mol})$ & $M_{w} / M_{n}$ & $\rho /\left(\mathrm{g} / \mathrm{cm}^{3}\right)^{\mathrm{a}, \mathrm{b}}$ & $G_{0} /(\mathrm{Pa})^{\mathrm{a}, \mathrm{b}}$ & $Z_{0}$ & $\tau_{e} /(\mathrm{s})$ \\
\hline Polyisoprene & $2.12 \times 10^{5}$ & 1.02 & 0.830 & $4.2 \times 10^{5}$ & 62.5 & $2.00 \times 10^{-4}$ \\
Polystyrene & $7.07 \times 10^{5}$ & 1.04 & 0.969 & $2.0 \times 10^{5}$ & 85.0 & $1.25 \times 10^{-2}$ \\
\hline \hline
\end{tabular}

${ }^{a}$ Reference 21.

${ }^{\mathrm{b}}$ At $413 \mathrm{~K}$.

$$
Z=M / M_{e} .
$$

However, the entanglement molecular weight $M_{e}$ of the primitive chain network model accounts for node fluctuations, and is therefore given by ${ }^{13}$

$$
M_{e}=\frac{1}{2} \frac{\rho R T}{G_{0}(\infty)} .
$$

It should also be noted that the primitive chain network model gives a power law exponent $\alpha$ to be used in Eq. (3) equal to $3.5 \pm 0.1 .^{3}$

\section{SIMULATIONS}

In this study, $G^{\prime}$ and $G^{\prime \prime}$ for monodisperse linear polymers were calculated and compared with existing data. Table I summarizes the relevant properties of the systems examined. ${ }^{19,20}$ The values of $Z$ in Table I were obtained from the experimental values of the plateau modulus through Eqs. (6) and (7). ${ }^{21}$

The calculations were performed with $\beta=1,1.8,3.5$, $6.5,11.8$ for polyisoprene, and $\beta=1,1.8,3.5,6.5,12.5,16.0$ for polystyrene (actual $\beta$ values are somewhat different because the average number of entanglements self-adjusts during the equilibration stage of the simulation). As usual, simulations use the average undilated subchain length $a$ as the unit of length, $k T$ as the unit of energy, and $\tau_{e}$ as the unit of time. For all calculations, the size of the simulation box was $12^{3}$, and the number density of subchains was 10 . Dynamic shear strain with strain amplitude of 0.25 was applied to the box, using Lees-Edwards boundary conditions. $G^{\prime}$ and $G^{\prime \prime}$ were obtained from the response after 5 complete cycles. The frequency range explored goes from $10^{-2}$ to 1 .

\section{RESULTS AND DISCUSSION}

Figure 1 shows $G^{\prime}$ and $G^{\prime \prime}$ for various $Z$, corresponding to the $\beta$ values previously indicated for polyisoprene. Shorter chains reveal terminal flow behavior in the low frequency region, and our previous study ${ }^{13}$ showed that the relaxation behavior of short chains is quantitatively in good agreement with experiments. Figure 1 also shows that, for longer chains, the calculated response corresponds to the rubbery plateau throughout the frequency range. This is consistent with our prediction that, for the longest chain, the frequency of terminal behavior would fall around $10^{-5}$, which is practically unattainable by direct simulation.

Figure 2 reports the same data of Fig. 1 shifted horizontally through the time scaling of Eq. (3), and vertically through the modulus scaling of Eq. (5). One should note that, because of the Rouse modes that follow a different scaling, the $G^{\prime \prime}$ curves show some deviations from a continuous curve in their high frequency regions.

Figure 3 is a comparison of the simulation results in Fig. 2 with the experimental data for the monodisperse linear polyisoprene melt of Ref. 19. From the nondimensional coordinates of Fig. 2 we go to the dimensional coordinates of Fig. 3 by using the values of modulus $G_{0}$ and of characteristic time $\tau_{e}$ reported in Table I. In Fig. 3 the irregularities introduced by the Rouse modes have been removed. Notice however that, except for the Rouse modes, the whole relaxation behavior of the long chain generated by the selfmultiscale scheme reproduces the experiments quantitatively, in a similar way as the direct calculations for short chains. ${ }^{13}$

It should be emphasized again that the long chain relaxation could not be simulated directly because of prohibitive computation time. Indeed, the calculation shown in Fig. 3 by the self-multiscale scheme was achieved in $17.5 \mathrm{~h}$ by a work-

(a)

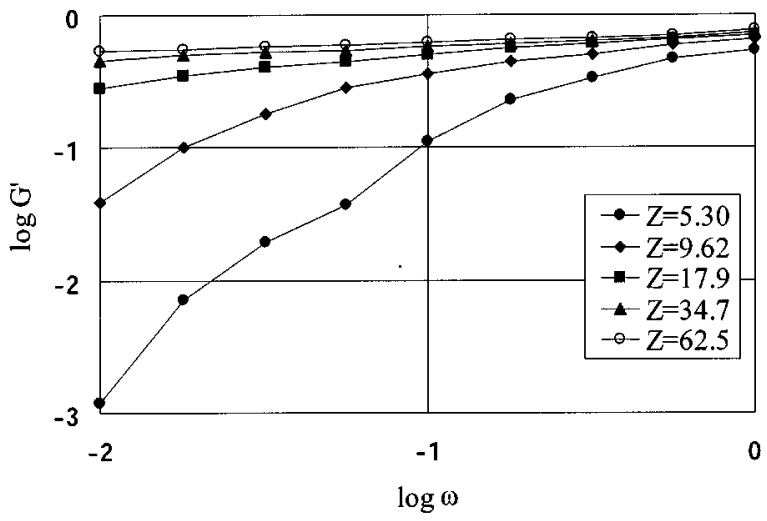

(b)

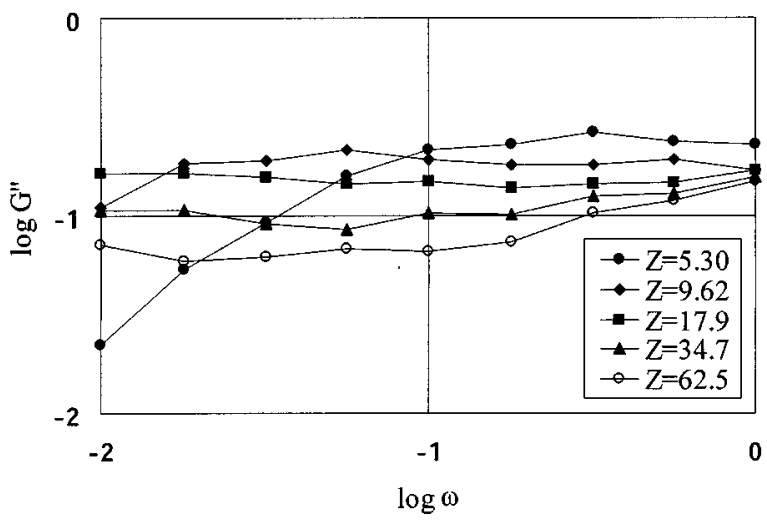

FIG. 1. (a) $G^{\prime}$ and (b) $G^{\prime \prime}$ for various $Z$ obtained by primitive chain network simulations. The frequency and modulus axes are made nondimensional (here and in Fig. 2) by using $\tau_{e}$ and $G_{0}(\infty)$, respectively. 
(a)

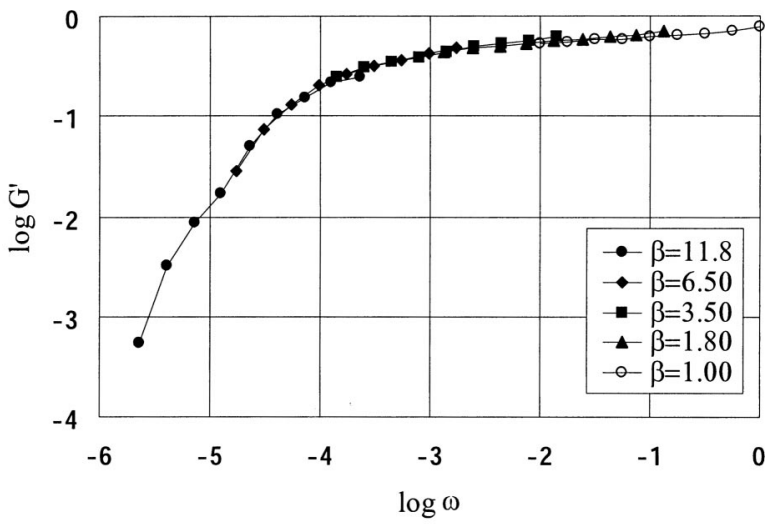

(b)

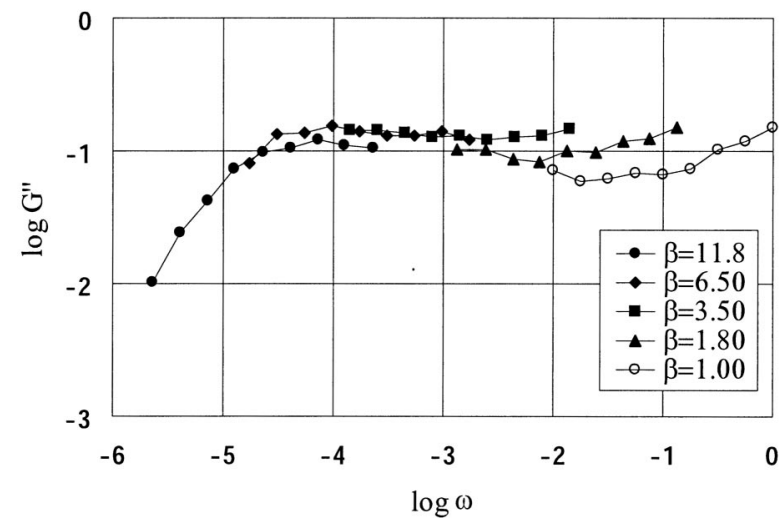

FIG. 2. (a) $G^{\prime}$ and (b) $G^{\prime \prime}$ for $Z=62.5$ chain generated from the data in Fig. 1 by the scaling rules given by Eq. (3) with $\alpha=3.4$, and Eq. (5).

station equipped with a Xeon $2.4 \mathrm{GHz}$ processor, while the estimated time for the direct calculation is around 7.6 $\times 10^{4} \mathrm{~h}(\sim 9$ years $)$.

Figure 4 shows the analogous results of the selfmultiscale calculation for the monodisperse polystyrene, compared with the experimental data from Ref. 20. As be-

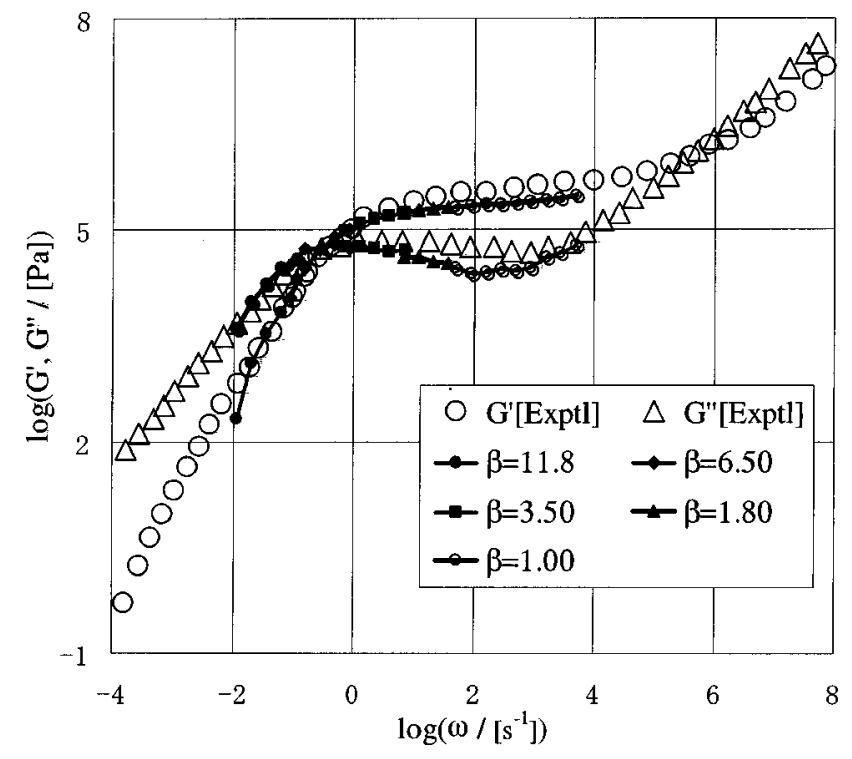

FIG. 3. Comparison of the results in Fig. 2 with data of a monodisperse linear polyisoprene melt (Ref. 19).

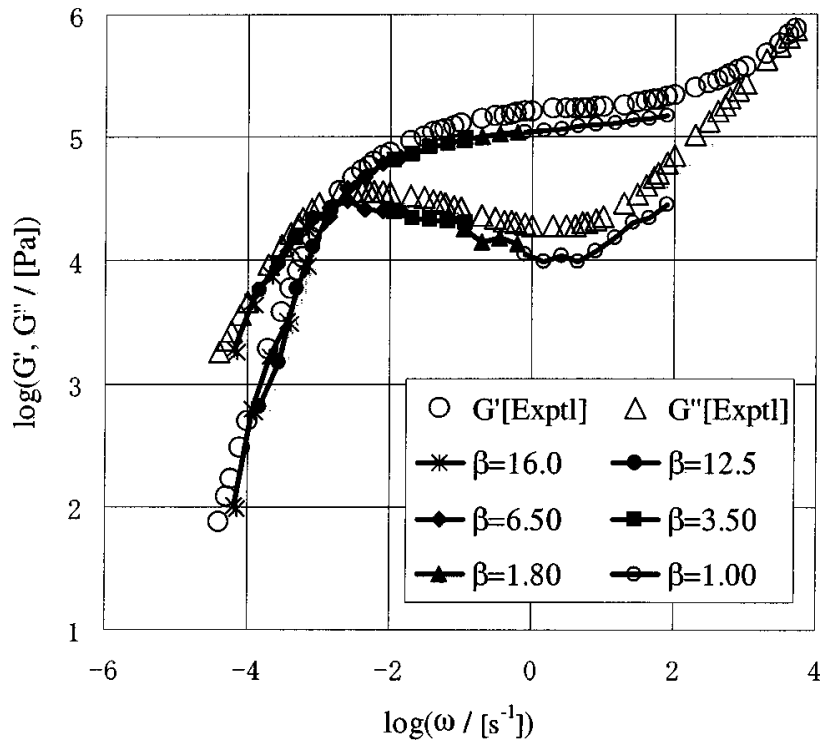

FIG. 4. Similar to Fig. 3 for the case of a monodisperse linear polystyrene melt (Ref. 20).

fore, the characteristic time and modulus used for data fitting are reported in Table I. Here again, the linear viscoelasticity is quantitatively captured.

\section{CONCLUSIONS}

Based on the idea of dynamic self-similarity as contained in the dynamic tube dilation picture, a calculation scheme was proposed for simulating the long time relaxation behavior of polymers with a large number of entanglements per chain. The scheme consists in computing the relaxation of a series of short chains in an easily accessible frequency window, from which the response of a long chain throughout a wide frequency range is obtained by suitable scaling factors for time and stress. These scaling laws are readily obtained from the DTD idea without any adjustable parameter.

The scaling law for time, being based on the terminal relaxation time of the chains, is not compatible with the Rouse time scaling law. Hence one disadvantage of the method is that it loses accuracy in the frequency (or time) range of the Rouse regime. However, that part of the response can be directly simulated on the full chain, i.e., before applying the DTD procedure.

The part of the relaxation spectrum which is not described at all by the model is that related to the chain segment between consecutive entanglements. That part of the chain is in fact replaced in the model by a fictitious phantom Gaussian spring.

Primitive chain network simulations were performed to test the scheme for linear monodisperse polyisoprene and polystyrene melts, which showed quantitative agreement with experiments.

Though the scheme proposed here is effective in reducing computational cost and in achieving calculations of interest to industry, it is fair to note that further study is required for polydisperse systems, for long chain branching, and for the nonlinear response under fast flows. Work is in progress in those directions. 


\section{ACKNOWLEDGMENTS}

The authors wish to acknowledge Mitsui Chemicals, Inc. for their permission to publish this paper. This study was supported in part by Grants in Aid for Scientific Research (KAKENHI) 2003 from the Ministry of Education, Culture, Sports, Science and Technology of Japan, and also by the "bio-rheo" project promoted by the Japan Science and Technology Agency.

\section{APPENDIX: SCALING FOR MODULUS}

To within a numerical factor of order unity, plateau modulus $G_{0}$ is written as follows:

$$
G_{0} \cong \frac{c b^{2}}{a^{2}} k T,
$$

where $b$ is monomer length, $a$ is subchain end-to-end distance, $c$ is the number density of monomers and $k T$ is thermal energy, respectively. ${ }^{6}$ Hence, for constant values of $c, b$, and $k T$, the plateau modulus $G_{0}$ scales with the subchain length $a$ as

$$
G_{0} \propto a^{-2} .
$$

In the DTD scheme, when in the course of relaxation the dilation ratio has matched the value of $\beta$, the subchain length has increased to the value

$$
a^{\prime}=\beta^{0.5} a .
$$

Hence, from Eq. (A2) we get

$$
G_{0}\left(\infty, a^{\prime}\right)=\frac{1}{\beta} G_{0}(\infty, a) .
$$

The modulus is here indicated with the $\infty$ symbol referring to the fact that the entanglement number chain-end effects are ignored. However, in the primitive chain network model, the plateau modulus $G_{0}(Z, a)$ for chains with $\mathrm{Z}$ segments accounts for chain-end effects through the correction

$$
G_{0}(Z) \equiv G_{0}(Z, a)=\left(1-\frac{3}{Z}\right) G_{0}(\infty, a) .
$$

After dilution, we can similarly write

$$
G_{0}\left(Z^{\prime}\right) \equiv G_{0}\left(Z^{\prime}, a^{\prime}\right)=\left(1-\frac{3}{Z^{\prime}}\right) G_{0}\left(\infty, a^{\prime}\right) .
$$

Hence,

$$
\frac{G_{0}\left(Z^{\prime}\right)}{G_{0}(Z)}=\frac{Z\left(Z^{\prime}-3\right)}{Z^{\prime}(Z-3)} \frac{G_{0}\left(\infty, a^{\prime}\right)}{G_{0}(\infty, a)} .
$$

Finally, by using the definition of $\beta$, Eq. (1), and Eq. (A4), Eq. (A7) reduces to Eq. (5) of the text.

${ }^{1}$ K. Kremer and G. S. Grest, J. Chem. Phys. 92, 5057 (1990).

${ }^{2}$ C. C. Hua and J. D. Schieber, J. Chem. Phys. 109, 10018 (1998).

${ }^{3}$ Y. Masubuchi, J.-I. Takimoto, K. Koyama, G. Ianniruberto, G. Marrucci, and F. Greco, J. Chem. Phys. 115, 4387 (2001).

${ }^{4}$ J.-I. Takimoto and M. Doi, Philos. Trans. R. Soc. London, Ser. A 361, 641 (2003).

${ }^{5}$ P. G. de Gennes, J. Chem. Phys. 55, 572 (1971).

${ }^{6}$ M. Doi and S. F. Edwards, The Theory of Polymer Dynamics (Clarendon, Oxford, 1986).

${ }^{7}$ W. W. Graessley, Adv. Polym. Sci. 47, 67 (1982).

${ }^{8}$ G. Marrucci, J. Non-Newtonian Fluid Mech. 62, 279 (1996).

${ }^{9}$ G. Marrucci, F. Greco, and G. Ianniruberto, J. Rheol. 44, 845 (2000).

${ }^{10}$ Y. Masubuchi, G. Ianniruberto, F. Greco, and G. Marrucci, Modell. Simul. Mater. Sci. Eng. 12, S91 (2004).

${ }^{11}$ G. Ianniruberto and G. Marrucci, J. Non-Newtonian Fluid Mech. 95, 363 (2000)

${ }^{12}$ Y. Masubuchi, H. Watanabe, G. Ianniruberto, F. Greco, and G. Marrucci, Nihon Reoroji Gakkaishi, J. Soc. Rheol., Jpn. 32, 197 (2004).

${ }^{13}$ Y. Masubuchi, G. Ianniruberto, F. Greco, and G. Marrucci, J. Chem. Phys. 119, 6925 (2003).

${ }^{14}$ G. Marrucci, J. Polym. Sci., Polym. Phys. Ed. 23, 159 (1985).

${ }^{15}$ T. C. B. McLeish, Adv. Phys. 51, 1379 (2002).

${ }^{16}$ H. Watanabe, Prog. Polym. Sci. 24, 1253 (1999).

${ }^{17}$ M. J. Struglinski and W. W. Graessley, Macromolecules 18, 2630 (1985).

${ }^{18}$ R. G. Larson, T. Sridhar, L. G. Leal, G. H. McKinley, A. E. Likhtman, and T. C. B. McLeish, J. Rheol. 47, 809 (2003).

${ }^{19}$ A. L. Frischknecht, S. T. Milner, A. Pryke, R. N. Young, R. Hawkins, and T. C. B. McLeish, Macromolecules 35, 4801 (2002).

${ }^{20}$ N. Golshan Ebrahimi, M. Takahashi, O. Araki, and T. Masuda, J. Rheol. 39, 1385 (1995).

${ }^{21}$ L. J. Fetters, D. J. Lohse, D. Richter, T. A. Witten, and A. Zirkel, Macromolecules 27, 4639 (1994). 